\title{
Diagnostic accuracy of F-18-fluoroethyltyrosine PET and PET/CT in patients with brain tumor
}

\author{
Vincent Dunet $\cdot$ John O. Prior
}

Received: 9 February 2013/Accepted: 18 March 2013/Published online: 6 April 2013

(C) Italian Association of Nuclear Medicine and Molecular Imaging 2013

\begin{abstract}
Positron emission tomography with ${ }^{18} \mathrm{~F}$-fluoroethyltyrosine (FET-PET) is increasingly used for the initial evaluation of patients with primary brain tumors (PBTs). This article provides an up-to-date systematic review and meta-analysis of FET-PET diagnostic performances in patients with suspected PBTs. The PubMed and EMBASE databases were searched for studies published in the period January 1977-September 2012. Inclusion criteria were: (1) use of FET-PET for assessment of newly diagnosed brain lesions; (2) no radiotherapy, surgery or chemotherapy prior to FET-PET and (3) use of histology as gold standard. A per-patient meta-analysis was performed, as well as a receiver operating characteristics (ROC) analysis of pooled patients to determine optimal tumor-tobackground ratio (TBR) of FET uptake. In total, 16 studies (641 patients) were included. For the diagnosis of tumoral vs non-tumoral brain lesions, FET-PET demonstrated a pooled sensitivity of 0.80 (95\% CI $0.74-0.85)$, specificity of $0.67(0.44-0.84)$, area under the curve of 0.81 (0.78-0.85), positive likelihood ratio of 2.4 (1.3-4.7), and negative likelihood ratio of $0.29(0.19-0.45)$. ROC analysis showed a meanTBR threshold $\geq 1.6$ and a maxTBR $\geq 2.2$ to have the best diagnostic value for differentiating PBTs from non-tumoral lesions. This meta-analysis confirms the excellent performances of FET-PET used for the diagnosis of PBTs. For FET-PET to become a relevant tool for
\end{abstract}

\footnotetext{
V. Dunet $\cdot$ J. O. Prior $(\bowtie)$

e-mail: john.prior@chuv.ch

V. Dunet

Department of Radiology, Lausanne

University Hospital, Rue du Bugnon 46,

1011 Lausanne, Switzerland
}

Department of Nuclear Medicine, Lausanne University

Hospital, Rue du Bugnon 46, 1011 Lausanne, Switzerland improving patient management, prospective multicenter studies with standardized acquisition protocols should investigate its added value over current magnetic resonance imaging and the optimal combination of FET-PET and MRI.

Keywords PET · F-18-fluoroethyltyrosine - Brain tumor · Glioma $\cdot$ Meta-analysis

\section{Introduction}

Primary brain tumors (PBTs) account for only 1-2 \% of adult cancers, according to the Central Brain Tumor Registry of the United States, with over 311,000 new patients with primary brain tumor or central nervous system tumor diagnosed between 2005 and 2009 [1]. Both tumoral lesions (gliomas, non-glioma PBTs, and metastases) and non-tumoral lesions (abscesses, parasitic lesions, demyelinating disease, infarcts, and old hematomas) may present as ring-enhancing masses on conventional magnetic resonance imaging (MRI) sequences. Gliomas, however, are the most frequent brain tumors and their histological differentiation and grading are predictive of aggressiveness and outcome [2]. They are currently divided into: (1) grade I and II tumors, considered low-grade with a protracted evolution, and (2) grade III (anaplastic glioma) and grade IV (glioblastoma) tumors, considered high-grade lesions leading to death within weeks to months when untreated.

Morphological assessment by MRI is very precise, allowing tumor diagnosis and assessment of tumor size, mass effect, edema and hemorrhagic effusion. However, it lacks specificity and does not easily allow the assessment of tumor activity and metabolism. Magnetic resonance spectroscopy (MRS), to increase specificity, investigates 
the presence of neuronal and membrane metabolites, such as $N$-acetylaspartate, choline, creatine, inositol or lactates, but it is limited by poor spatial resolution and frequent artifacts due to proximity with spinal fluid or skull bone. On the contrary, molecular imaging with positron-emission tomography (PET) provides information on tumor metabolism and grade, and helps to identify and delineate tumor zones with increased growth activity [3]. As PET with ${ }^{18} \mathrm{~F}$ fluorodeoxyglucose (FDG) is not able to reliably predict the tumoral nature of a lesion due to high uptake in normal brain and unspecific uptake in inflammatory or benign lesions, amino acid tracers have been developed in recent decades.

${ }^{18} \mathrm{~F}$-fluoroethyltyrosine (FET) is an artificial amino acid which is taken up into upregulated tumoral cells via the membranous $\mathrm{L}_{-}, \mathrm{B}^{0,+}$ - and $\mathrm{B}^{0}$-transporter system and accumulates in the cell; it is not incorporated into proteins like the natural amino acids, such as ${ }^{11} \mathrm{C}$-methionine, and its uptake is not directly proportional to grade [4, 5]. However, FET can be used to obtain good contrast images in both high- and low-grade tumors, at a lower effective dose as compared to FDG [6, 7]. Qualitative FET-PET uptake does not allow tumor grading, but dynamic FETPET analysis helps in the differentiation of low- from highgrade tumors [8]. FET-PET has also been shown to be valuable for guiding biopsy, diagnosing brain tumor recurrence after initial therapy, and directing radiosurgery [9]. As FET exhibits lower uptake by inflammatory cells than ${ }^{11} \mathrm{C}$-methionine and FDG, it can be used for differentiating tumor from radionecrosis [10]. Early changes in FET uptake have also been shown to be helpful in predicting tumor response to treatment and assessing prognosis [11]. As there are only a few published studies with limited patient populations that evaluate FET-PET in newly identified brain lesions, a couple of years ago, we carried out a systematic review and meta-analysis which demonstrated excellent performances of FET-PET for the diagnosis of PBT and glioma [12].

The purpose of this report is to provide an up-to-date systematic review and meta-analysis of the diagnostic performances of FET-PET in patients with suspicion of primary brain tumors and gliomas and to assess the performance of FET uptake quantification.

\section{Materials and methods}

Data sources and search

We performed a systematic literature search of the PubMed and EMBASE medical databases, looking for English and non-English publications from the period January 1977 to September 2012. As previously reported [12], the search terms were: "("O-(2-fluoroethyl)tyrosine"[All fields] OR " $\left({ }^{18} \mathrm{~F}\right)$ fluoroethyltyrosine" [All fields] OR "Tyrosine/diagnostic use"[Mesh]) AND ("Brain Neoplasms/radionuclide imaging"[Mesh] OR "Glioma"[All fields]) AND "Humans"[Mesh])". Errata, reviews, preclinical, animal and non-radiopharmaceutical studies were excluded.

Study selection

We considered only human studies in which FET-PET was used in the assessment of newly diagnosed, untreated brain tumors. Inclusion criteria were: (1) FET-PET was used in patients with newly diagnosed brain lesions; (2) patients had received no radiotherapy, surgery or chemotherapy prior to FET-PET, and (3) histology was used as the gold standard. Studies written in languages other than English, French, or German were excluded, as were ones that included fewer than ten patients.

\section{Data extraction and quality assessment}

From each study, we collected the following information: first author, year of publication, study population (number of patients with initial assessment of brain tumor, gender, age, and histological analysis); FET-PET results [positive or negative examination as assessed by authors, maximal target-to-background ratio (TBR) and/or mean TBR, when available] and PET imaging protocol (time between injection and image acquisition, static or dynamic imaging protocol, and number of frames). Data at patient level, when provided, were recorded. Study quality and applicability were assessed using the Quality Assessment of Diagnostic Accuracy Studies checklists (QUADAS, scale range 0-14) and the Standards for Reporting Studies of Diagnostic Accuracy checklist (STARD, scale range 0-25) $[13,14]$.

\section{Statistical analysis}

We performed all analyses at patient level using dichotomized histological diagnosis (tumor or not, glioma or not) as the gold standard according to the WHO classification of tumors of the central nervous system [2] and the 3rd edition of the International Classification of Diseases for Oncology (ICD-O-3); glioma was defined by ICD-O-3 codes 9380-9384, 9391-9460, and 9480. We used the criteria described in each study to define FET-PET positivity. Data synthesis used a bivariate mixed-effects regression model and average sensitivity, specificity, positive and negative likelihood ratios (LRs) and odds ratios with their respective $95 \%$ confidence intervals (CIs) were calculated from the maximum likelihood estimates and graphically assessed by a summary receiver operating characteristics (SROC) 
curve with computation of the area under the curve (AUC). SROC is a composite statistic reflecting the discriminative ability of a diagnostic test and it can overcome some limitations associated with the direct pooling of sensitivity and specificity values of individual studies [15]. We used graphical analysis of Forest plots and statistical analysis with the $\chi^{2}$ and Cochran's $Q$ tests to assess heterogeneity of results. Inconsistency of between-study results was assessed using the $I^{2}$ statistics (percentage of total variation across studies attributable to heterogeneity rather than chance). The Funnel plot asymmetry test assessed publication bias (tendency to publish only positive results) [16]. This was performed using the regression of logarithmic diagnostic odds ratio against the inverse square root of the effective sample size (ESS) weighted by ESS with a $p$ value
$<0.05$ for the slope coefficient indicating significant asymmetry [17].

We also carried out analyses at patient level to determine the optimal diagnostic threshold of mean TBR (meanTBR $=$ mean activity of the lesion/mean activity of the contralateral brain) and maximum TBR (max$\mathrm{TBR}=$ max activity of the lesion/mean activity of the contralateral brain). In the studies, a variety of methods were used for computing TBR: (1) a region-of-interest (ROI) manually drawn around the tumor based on T1- and/ or T2-weighted MR images [18-20], or (2) increased FET uptake [8, 21-24], (3) automated ROI drawing of the area with increased FET uptake $>3$ standard deviations of the mean standardized uptake value (SUV) of a control region [25], or (4) based on a $90 \% \mathrm{SUV}_{\max }$ threshold isocontour
Fig. 1 Flowchart of study identification and selection. *Note: Among these, a subpopulation of $n=14$ studies with $N=525$ patients did report target-to-background ratio (TBR) data, which were used for deriving TBR thresholds yielding the best diagnostic performances

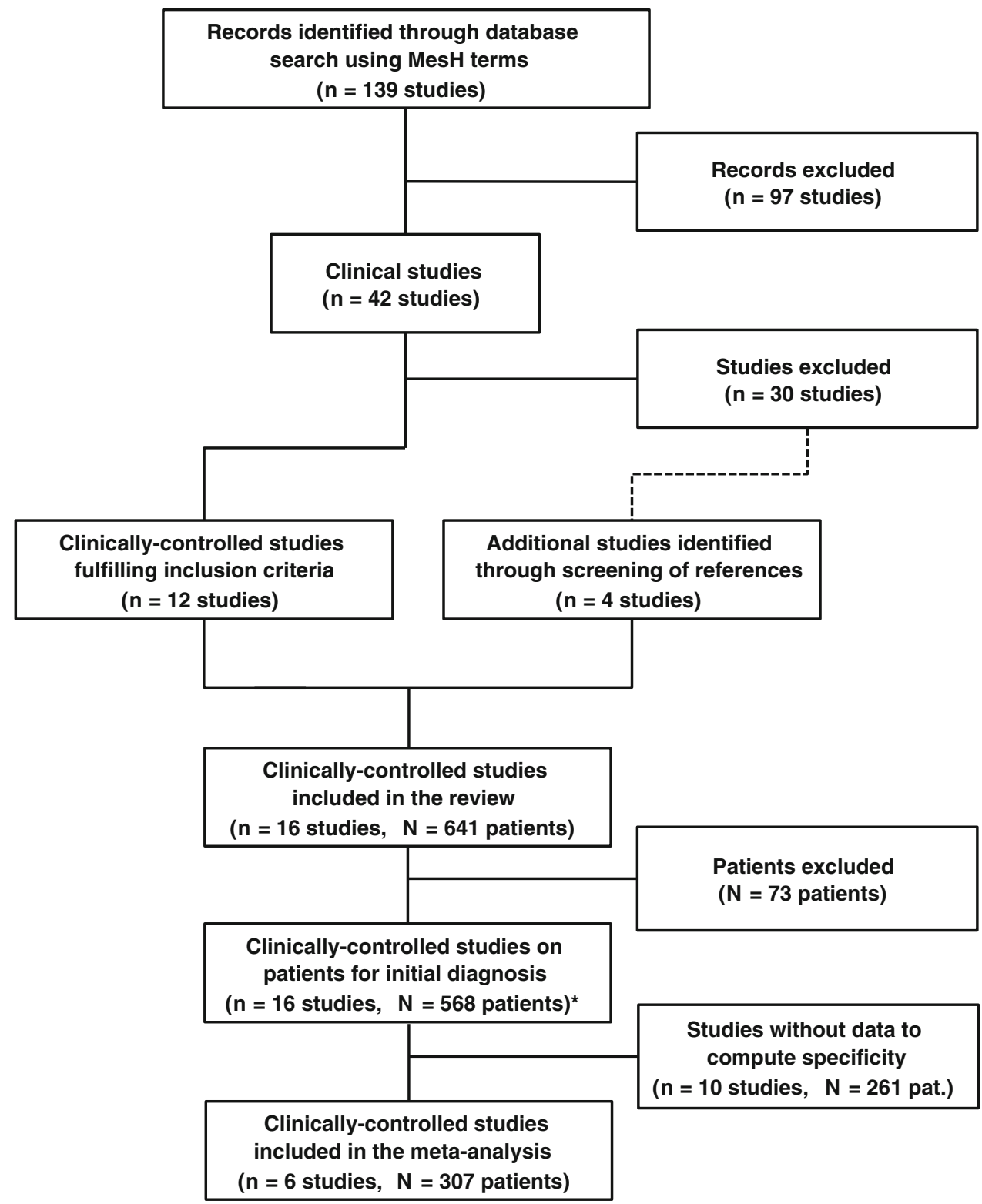


method [26], or (5) a fixed 25-mm ${ }^{2}$ elliptic ROI centered on the area of most increased FET uptake [27, 28]. In three studies, the method used to define the ROI was not specified [29-31]. We performed receiver operating characteristics (ROC) analysis of pooled individual patient values to determine the optimal meanTBR and maxTBR thresholds. Finally, Student's $t$ test was used to assess the difference in meanTBR or maxTBR according to glioma WHO grade. All statistics were performed with Stata 12.1 (StataCorp, College Station, TX, USA). $p$ values $<0.05$ were considered to indicate statistical significance. By convention throughout the text, tables and figures, we used the small letter $n$ to describe the number of studies and the capital letter $N$ to describe the number of patients.

\section{Results}

\section{Study characteristics}

Our initial search of the PubMed and EMBASE databases identified a total of $n=139$ articles. After exclusion of non-FET reports $(n=78)$, errata $(n=2)$, reviews $(n=10)$, and preclinical $(n=6)$ and animal studies $(n=1), 42$ clinical articles on FET-PET in patients remained (Fig. 1). After applying the inclusion criteria, 12 studies remained, excluding reports on recurrent disease $(n=18)$, reports without FET results $(n=4)$, reports including $<10$ primary brain lesions $(n=4)$, reports on non-tumoral brain lesions $(n=3)$, and our previous meta-analysis $(n=1)$ [12]. Four additional studies were found through reference screening of the excluded papers (Fig. 1).

Sixteen studies [8, 18-32] with a total of $N=641$ patients (Table 1) were eligible for inclusion in the present metaanalysis. Six studies had mixed populations with both primary brain tumor and recurrence $(\# 1,9,11,12,13$, and 14, Table 1) and one did not report histology results for all patients (\#2, Table 1). Consequently, from these seven studies only patients with FET-PET results in primary assessment of the lesion verified by a subsequent biopsy or resection were included. Thus, $N=568$ patients finally remained (median age 45 years; mean age $45 \pm 15$ years; sex ratio $\mathrm{F}: \mathrm{M}=$ 0.8:1), of whom $516(91 \%)$ had a tumor, which was a glioma in $N=498(88 \%)$ patients. Non-glioma brain tumor was diagnosed in $N=18$ patients $(N=5$ lymphomas, $N=4$ metastases, $N=3$ hemangioblastomas, $N=1$ cavernoma, $N=1$ germinoma, $N=1$ teratoma, $N=1$ medulloblastoma, $N=1$ pinealoblastoma, and $N=1$ primitive neuroectodermal tumor). There were also $N=52$ non-malignant lesions $(N=22$ gliosis lesions, $N=11$ infectious disease lesions, $N=5$ demyelinating lesions, $N=4$ infarcts, $N=4$

Table 1 Characteristics of the studies

\begin{tabular}{|c|c|c|c|c|c|c|c|c|c|}
\hline$n$ & Study & Years & $\begin{array}{l}\text { Patients } \\
N\end{array}$ & $\begin{array}{l}\text { Excluded } \\
N(\%)\end{array}$ & $\begin{array}{l}\text { Age } \\
\text { (years) }\end{array}$ & $\begin{array}{l}\text { Sex ratio } \\
\text { (W:M) }\end{array}$ & $\begin{array}{l}\text { Tumour } \\
N(\%)\end{array}$ & $\begin{array}{l}\text { Glioma } \\
N(\%)\end{array}$ & $\begin{array}{l}\text { Time } \\
(\mathrm{min})\end{array}$ \\
\hline $1^{\mathrm{a}}$ & Pauleit et al. [21] & 2004 & 20 & $6(30)$ & $53 \pm 11$ & $12: 2$ & $12(60)$ & $11(55)$ & $15-10$ \\
\hline 2 & Weckesser et al. [25] & 2005 & 44 & $9(20)$ & $45 \pm 22$ & $15: 20$ & $31(70)$ & $24(55)$ & $30-10$ \\
\hline 3 & Pauleit et al. [27] & 2005 & 28 & $0(0)$ & $42 \pm 20$ & $19: 9$ & $23(82)$ & $23(82)$ & $15-10$ \\
\hline $4^{\mathrm{a}}$ & Floeth et al. [29] & 2005 & 50 & $0(0)$ & $44 \pm 17$ & $29: 21$ & $34(68)$ & $34(58)$ & $15-10$ \\
\hline $5^{\mathrm{a}}$ & Floeth et al. [18] & 2006 & 14 & $0(0)$ & $55 \pm 12$ & $1: 13$ & $5(36)$ & $5(36)$ & $15-10$ \\
\hline 6 & Popperl et al. [8] & 2007 & 54 & $0(0)$ & $49 \pm 17$ & $26: 28$ & $54(100)$ & $54(100)$ & $20-10$ \\
\hline 7 & Floeth et al. [19] & 2007 & 33 & $0(0)$ & $41 \pm 9$ & $13: 20$ & $33(100)$ & $33(100)$ & $15-10$ \\
\hline 8 & Stockhammer et al. [30] & 2008 & 22 & $0(0)$ & $43 \pm 13$ & $13: 9$ & $22(100)$ & $22(100)$ & $10-30$ \\
\hline $9^{\mathrm{a}}$ & Pauleit et al. [20] & 2009 & 52 & $10(19)$ & $46 \pm 13$ & $16: 26$ & $34(65)$ & $33(65)$ & $30-50$ \\
\hline 10 & $\begin{array}{l}\text { Benouaich-Amiel et al. } \\
\text { [31] }\end{array}$ & 2010 & 12 & $0(0)$ & - & - & $12(100)$ & $11(92)$ & - \\
\hline $11^{\mathrm{a}}$ & Pichler et al. [22] & 2010 & 88 & $28(32)$ & - & - & $59(67)$ & $51(59)$ & $30-50$ \\
\hline 12 & Plotkin et al. [32] & 2010 & 15 & $4(27)$ & $44 \pm 12$ & $7: 4$ & $11(73)$ & $11(73)$ & $10-10$ \\
\hline 13 & Floeth et al. [28] & 2011 & 30 & $4(13)$ & $42 \pm 11$ & $9: 17$ & $26(87)$ & $26(87)$ & $15-15$ \\
\hline 14 & Calcagni et al. [23] & 2011 & 32 & $12(38)$ & $42 \pm 16$ & $6: 14$ & $20(100)$ & $20(100)$ & $5-65$ \\
\hline 15 & Jeong et al. [24] & 2012 & 20 & $0(0)$ & $52 \pm 17$ & $7: 13$ & $20(100)$ & $20(100)$ & $30-50$ \\
\hline $16^{\mathrm{a}}$ & Jansen et al. [26] & 2012 & 127 & $0(0)$ & $46 \pm 14$ & $55: 72$ & $120(94)$ & $120(94)$ & $20-40$ \\
\hline \multicolumn{3}{|c|}{ Meta-analysis subtotal } & 351 & $44(13)$ & $46 \pm 15$ & $113: 134$ & $264(75)$ & $254(72)$ & - \\
\hline \multicolumn{3}{|c|}{ Overall total } & 641 & $73(11)$ & $46 \pm 15$ & $270: 298$ & $516(80)$ & $498(78)$ & - \\
\hline
\end{tabular}

Results 1-12 were originally published in Ref. [12] (c) by the Society of Nuclear Medicine and Molecular Imaging, Inc

a Included in the meta-analysis 
hemorrhages, $N=2$ encephalitis, $N=2$ cortical dysplasia, $N=1$ adenoma, and $N=1$ aneurysm).

Performance for the diagnosis of brain tumor and glioma

Six of the 16 selected studies, for a total of $N=307$ patients, could be included in the bivariate mixed-effects regression model (Fig. 1). The other ten studies could not be included in our analysis as they (1) did not report the true negative and false positive rates necessary to compute specificity, or (2) did not report dichotomized FET-PET results. The results of the pooled analysis showed, for FET-PET diagnosis of tumoral vs

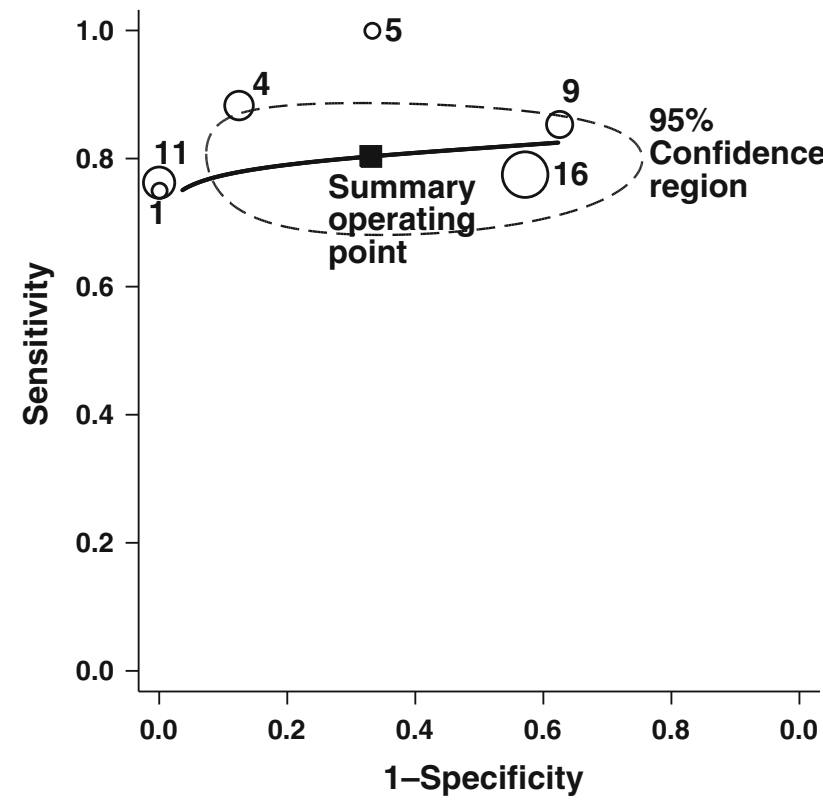

Fig. 2 Summary receiver operating characteristics (SROC) curve for the diagnosis of tumoral vs non-tumoral brain lesions non-tumoral brain lesions, a sensitivity of $0.80(95 \% \mathrm{CI}$ $0.74-0.85)$, a specificity of $0.67(0.44-0.84)$, a positive LR (LR+) of 2.4 (1.3-4.7), a negative LR (LR-) of 0.29 (0.19-0.45), a diagnostic odds ratio of 8 [3-23], and an AUC of $0.81(0.78-0.85)$. The SROC curve is presented in Fig. 2 with study symbols weighted by patient population size.

Similarly, FET-PET diagnosis of glioma vs non-glioma brain lesions showed a sensitivity of $0.82(0.74-0.87)$, a specificity of $0.59(0.32-0.81)$, an LR+ of $2.0(1.0-3.9)$, an LR - of 0.31 (0.16-0.61), a diagnostic odds ratio of 6 [2-23], and an AUC of $0.82(0.78-0.85)$.

Assessment of the heterogeneity, inconsistency, and quality of the studies

Forest plots (Fig. 3) did not show any significant performance heterogeneity or inconsistency between studies for the diagnosis of brain tumors $(Q=1.5, p=0.24$, and $I^{2}=0 \%$ ). Likewise, glioma diagnosis did not reveal significant performance heterogeneity $(Q=3.8$, $p=0.08)$ or inconsistency $\left(I^{2}=47 \%\right)$, although specificity differed significantly between studies $(Q=15.8$, $p=0.01$ ). Funnel plots (Fig. 4a) did not reveal any publication bias for brain tumor $(p=0.64)$, or glioma diagnosis $(p=0.98)$. Figure $4 \mathrm{~b}$ summarizes graphically the assessment of study quality using the QUADAS and STARD criteria.

Quantitative tumor-to-background ratio (TBR) threshold for diagnosing brain tumor and glioma

Individual patient meanTBR results were given in $n=9$ studies $(N=271$ patients) $[18-22,27-29,31])$ and maxTBR in $n=7$ studies ( $N=329$ patients) [8, 19, 20, 24-26, 30]. The ROC curve analysis indicated that meanTBR $\geq 1.6$
Fig. 3 Forest plots of sensitivity and specificity for brain tumor diagnosis of the $n=6$ studies included in the meta-analysis
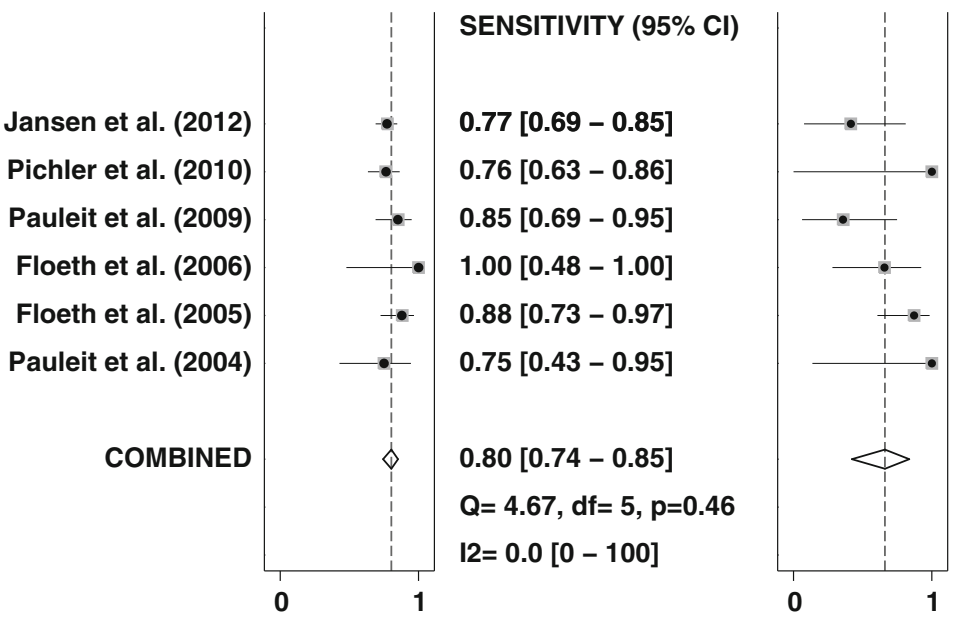

SPECIFICITY $(95 \% \mathrm{Cl})$

$0.43[0.10-0.82]$

$1.00[0.03-1.00]$

$0.38[0.09-0.76]$

$0.67[0.30-0.93]$

$0.88[0.62-0.98]$

1.00 [0.16 - 1.00]

0.67 [0.44 - 0.84]

$Q=9.48, d f=5, p=0.09$

$12=47.3[0-96]$ 

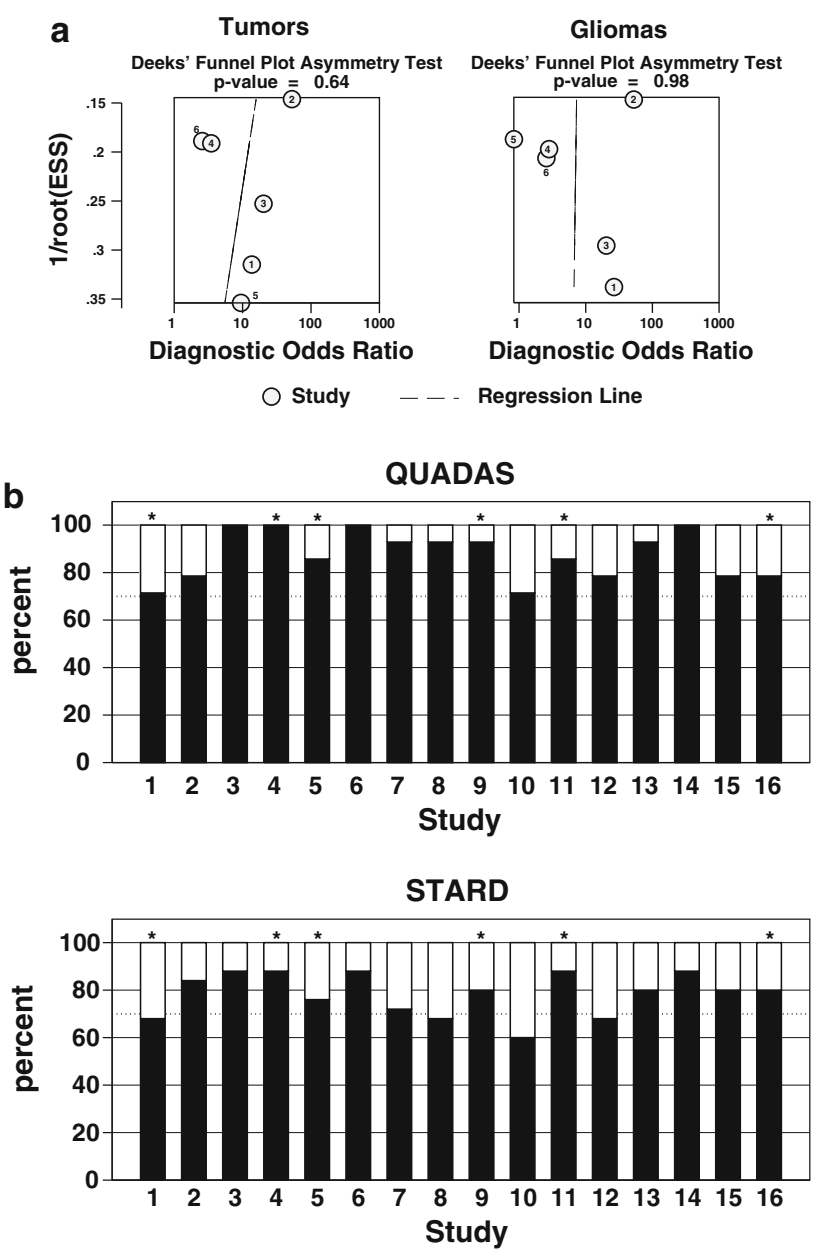

Fig. 4 a Funnel plots of the inverse square root of the effective sample size (ESS) vs diagnostic logarithmic odds ratio showing no significant publication bias for tumors or gliomas. b Study quality grading using QUADAS (range 0-14) and STARD scores (range 0-25). Asterisk these studies were included in the meta-analysis

and $\operatorname{maxTBR} \geq 2.2$ had the best value for diagnosing tumoral vs non-tumoral brain lesions, with a 0.77 (0.71-0.82) sensitivity, $0.81(0.64-0.92)$ specificity, 0.96 (0.92-0.98) positive predictive value, and $0.35(0.25-0.46)$ negative predictive value for meanTBR, and a 0.65 (0.60-71) sensitivity, $0.82 \quad(57-96)$ specificity, 0.99 (96-1.0) positive predictive value, and $0.11(0.06-0.19)$ negative predictive value for maxTBR. For diagnosing brain glioma vs non-glioma brain disease, meanTBR $\geq 1.7$ and $\operatorname{maxTBR} \geq 2.2$ showed the best diagnostic performances, having sensitivity, specificity, positive predictive and negative predictive values of $0.71(0.65-0.77), 0.72$ (0.57-0.84), 0.92 (0.87-0.96), $0.34(0.25-0.45)$, and 0.65 $(0.60-0.71), 0.67 \quad(0.45-0.84), 0.96 \quad(0.93-0.98), 0.13$ (0.08-0.20), respectively.

High-grade gliomas (WHO I-II) had significantly higher meanTBR and maxTBR values than the low-grade gliomas

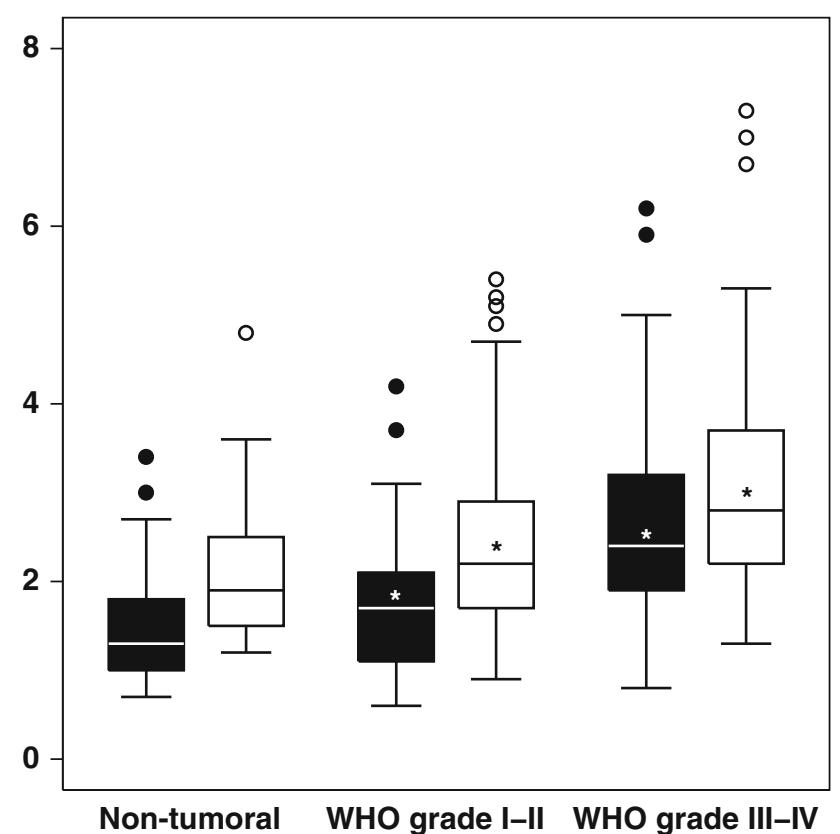

Fig. 5 Target-to-background (TBR) comparison box plot according to histological WHO grading (black box meanTBR, white box maxTBR). ${ }^{*} p<0.001$ vs non-tumoral lesions

(WHO III-IV), and non-tumoral lesions ( $2.6 \pm 1.0$ vs $1.7 \pm 0.7$ vs $1.5 \pm 0.6, p<0.001 ; 3.0 \pm 1.1$ vs $2.4 \pm 1.0$ vs $2.1 \pm 0.8, p<0.001$ ) (Fig. 5). There was no significant difference in TBR between non-tumoral lesions and lowgrade gliomas $(p<0.3)$.

Fig. 6 a T1-weighted sagittal view (left) in a 47-year-old patient with a growing anterior frontal mass with decreased T1-weighted and enhanced T2-weighted (right) signal, with restricted diffusion, perfusion with neoangiogenesis signs, and no Gd-enhancement. Spectroscopy showed a tumoral spectrum with decreased $\mathrm{N}$-acetylaspartate (NAA) and an increased choline/creatine ratio of 2 . The most probable diagnosis was intermediate-grade oligodendroglioma of corresponding ${ }^{18}$ F-FET-PET fused with T1-weighted image (bottom) showed an increased tumor-to-background ratio (TBR) of

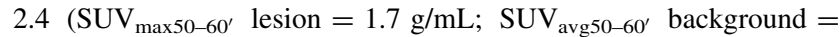
$0.7 \mathrm{~g} / \mathrm{mL}$ ) with a washout indicating high-grade glioma. The final diagnosis was grade III anaplastic oligoastrocytoma. b T1-weighted sagittal view (left) in a 73-year-old patient with a corpus callosum and right cingular gyrus lesion with decreased T1-weighted and enhanced T2-weighted (right) signal, with restricted diffusion and perfusion with neoangiogenesis signs, and no Gd-enhancement. The likely diagnosis was intermediate-grade glioma. The ${ }^{18} \mathrm{~F}$-FET-PET/T1weighted MR image (bottom) showed an increased TBR of 2.2 $\left(\mathrm{SUV}_{\max 50-60^{\prime}}\right.$ lesion $=1.8 \mathrm{~g} / \mathrm{mL} ; \mathrm{SUV}_{\text {avg50-60' }}$ background $=0.8 \mathrm{~g} /$ $\mathrm{mL}$ ) with a radiotracer washout indicating high-grade glioma. The final diagnosis was grade III anaplastic astrocytoma. For both lesions, the information gained by ${ }^{18} \mathrm{~F}$-FET helped to direct the biopsy to the area of highest TBR and pinpointed higher-grade (III-IV) washout curves. None of these lesions had any Gd-enhancement on MR, making it difficult to select the biopsy target 

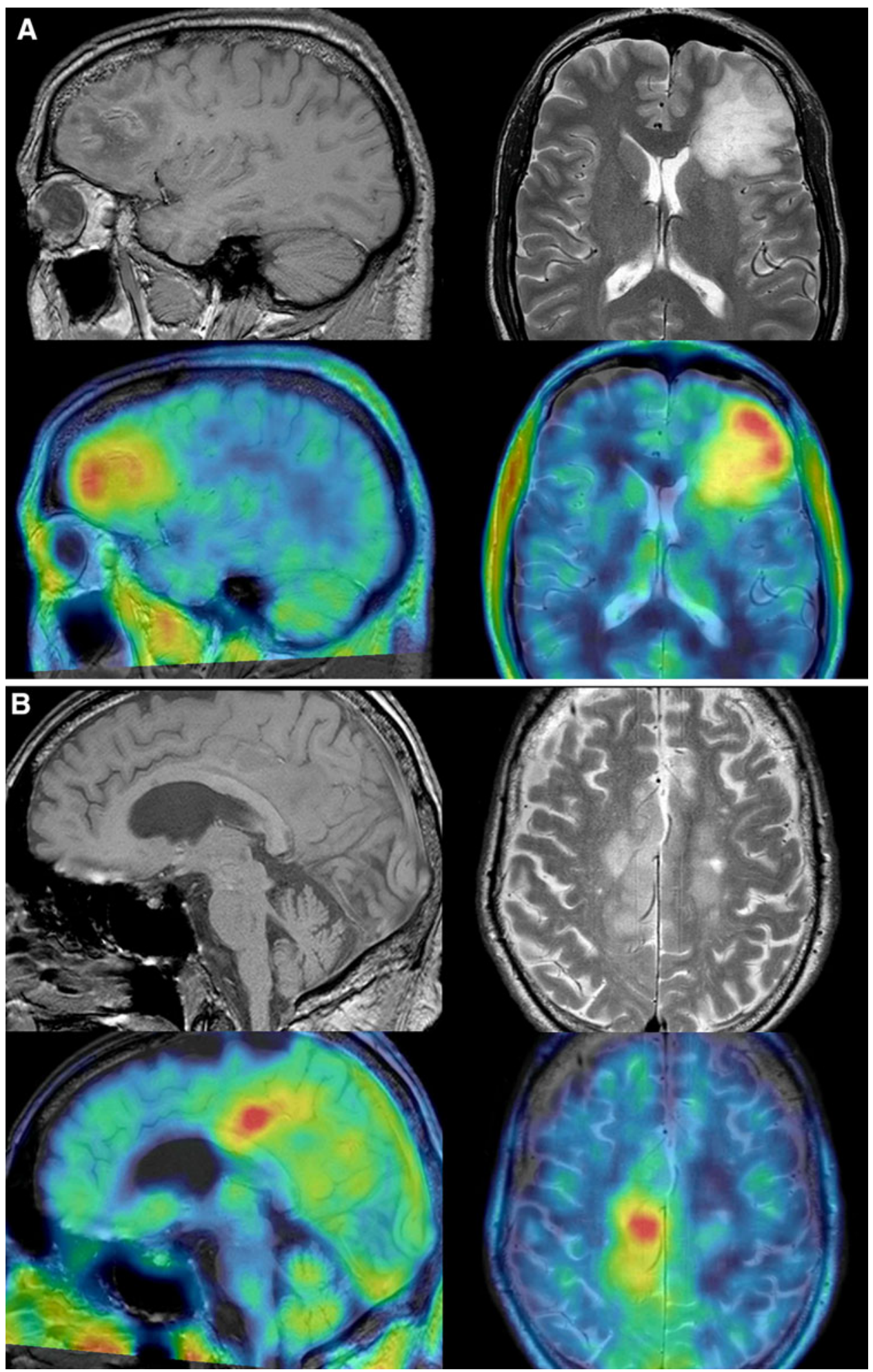

\section{Discussion}

Our updated systematic review and meta-analysis confirms the good performances of FET-PET for the initial diagnosis of brain tumors and gliomas. In this large population of patients with newly diagnosed brain lesions, FET was found to have good sensitivity ( 80 and $82 \%$ ) and moderate specificity (67 and $59 \%$ ) for the diagnosis of brain tumors and gliomas, respectively. The high LR + and low LRsuggest that FET-PET is useful for confirming or excluding 
brain tumors or gliomas. The high positive predictive value of the proposed mean- and maxTBR thresholds confirms the utility of quantitative analyses for identifying brain tumors and gliomas.

For over a decade, FET-PET has been known to help in distinguishing between proliferating tumoral and nontumoral lesions [5, 10]. An initial PET comparison study demonstrated that FET uptake ratios correlated with ${ }^{11} \mathrm{C}$ methionine tumor uptake, with the advantage that FETPET does not require an on-site cyclotron [6]. This allowed more centers to have access to FET. Comparison studies also showed lower uptake by inflammatory cells [33-35], opening the way for better discrimination between infectious and tumoral lesions [36], and between recurrent tumor and radionecrosis [10]. Following these early studies in recurrent tumor [37], several studies assessed FET-PET performances in the initial evaluation of isolated brain tumors. Of the sixteen studies included in this systematic review, only six provided enough details to determine sensitivity and specificity, suggesting that there is a need for better reporting of FET-PET study results. By comparison, MRI had a diagnostic accuracy of $55 \%$, which increased to $71 \%$ when MRS was added in one large study ( $N=176$ ) [38], a value that is still lower than the AUC of $0.81(0.78-0.85)$ reported in this updated meta-analysis. This finding confirms our previous results that seemed to show that the ability of FET-PET to assess the tumoral nature of isolated brain lesions was superior to that of MRI alone [12]. This is also known from specific studies combining PET and MR examinations, such as the one by Pauleit et al. [27] in which PET-MR fusion significantly increased the specificity for guiding diagnostic biopsy from $53 \%$ for MRI alone to $94 \%$ (Fig. 6). In another study, Floeth et al. [29] reported that the combination of MRI with FET-PET and MRS yielded a diagnostic accuracy of $97 \%$.

Quantitative imaging analysis using ratio to normal brain (meanTBR and maxTBR) is useful for characterizing lesion uptake and allows inter- and intra-patient comparisons, as normal brain FET uptake can be quite variable on consecutive PET examinations, even in the same patient. As in our previous meta-analysis [12], meanTBR $\geq 1.6$ and maxTBR $\geq 2.2$ thresholds demonstrated excellent performance for diagnosing brain tumor. This is consistent with results previously reported by Pauleit et al. [27] (meanTBR $\geq 1.6$ for tumoral tissue) and by Pöpperl et al. [37] (maxTBR $>2.0$ for recurrence). Similarly, for gliomas, a meanTBR $\geq 1.7$ and a maxTBR $\geq 2.2$ demonstrated excellent diagnostic performances, with significantly lower meanTBR and maxTBR in low-grade than in high-grade gliomas. On dynamic acquisitions, several studies $[8,23$, 26, 39] reported accurate distinction between low-grade and high-grade untreated gliomas using information from tumor time-activity curves, such as an increasing/decreasing slope uptake pattern $[26,39]$ or early-to-middle uptake ratio [23]. Whether combining conventional MRI with MRS, diffusion imaging and dynamic FET-PET imaging could provide better performances for diagnosing glioma that has not been established in large populations [40, 41]. This could also be an interesting issue to explore in combinations with the latest hybrid PET/MRI scanners [42].

Although we were able to include only 16 studies in our analysis, they were of good quality with 16/16 (100\%) having QUADAS scores $\geq 10$ and 12/16 (75\%) STARD scores $\geq 18$, and they all used a histological WHO classification gold standard. Moreover, there was no publication bias or inconsistency between the studies, strengthening the validity of our results. Nonetheless, some limitations remain. First, the meta-analysis included only $n=6$ studies, due to patient population (absence of true negative cases) or methodological (absence of dichotomized reporting of FET-PET results) limitations. Second, the optimal TBR thresholds identified from our meta-analysis must be interpreted cautiously, as these values are PET protocol-dependent. For instance, differences in uptake kinetics between low- and high-grade tumors exist, and these could influence TBR, particularly if early (10-20 min post-injection) frames are added to late $>30$-min frames $[8$, 25]. Moreover, the use of several methods to define regions of interest most certainly influences TBR.

Further investigations are needed and great care should be taken to ensure strict standardization of PET acquisition protocols to increase FET-PET inter-center reproducibility and allow multicenter data analysis. Recommendations on imaging protocols already exist, such as the EANM and German guidelines on amino acid analogs for PET imaging of the brain $[43,44]$. The inclusion of more true negative cases would also help to better determine the specificity, although this could be difficult, as patients may not necessarily undergo biopsy if FET-PET is negative. Finally, although cost-effectiveness of FET-PET in the management of patients with brain tumor has recently been studied, the results obtained should be treated with caution due to several limitations of the reported model $[45,46]$. Thus, there is still a need for cost-effectiveness studies of FETPET, used separately or in combination with MRI, in the clinical setting.

\section{Conclusion}

The results of this updated systematic review and metaanalysis confirm the good performances of FET-PET for the initial assessment of patients with new, isolated, untreated brain lesions. Strict standardization of PET imaging protocols and large, prospective, multicenter 
studies investigating the added value of this technique over conventional MRI and new MRI methods are now needed to consolidate FET-PET as a highly relevant tool for patient management.

Conflict of interest The authors do not declare any conflict of interest.

\section{References}

1. Dolecek TA, Propp JM, Stroup NE, Kruchko C (2012) CBTRUS statistical report: primary brain and central nervous system tumors diagnosed in the United States in 2005-2009. Neuro Oncol 14(Suppl 5):v1-v49. doi:10.1093/neuonc/nos218

2. Louis DN, Ohgaki H, Wiestler OD, Cavenee WK, Burger PC, Jouvet A, Scheithauer BW, Kleihues P (2007) The 2007 WHO classification of tumours of the central nervous system. Acta Neuropathol 114(2):97-109. doi:10.1007/s00401-007-0243-4

3. la Fougere C, Suchorska B, Bartenstein P, Kreth FW, Tonn JC (2011) Molecular imaging of gliomas with PET: opportunities and limitations. Neuro Oncol 13(8):806-819. doi:10.1093/ neuonc/nor054

4. Heiss P, Mayer S, Herz M, Wester HJ, Schwaiger M, Senekowitsch-Schmidtke R (1999) Investigation of transport mechanism and uptake kinetics of $O$-(2-[18F]fluoroethyl)-L-tyrosine in vitro and in vivo. $\mathrm{J}$ Nucl Med 40(8):1367-1373

5. Wester HJ, Herz M, Weber W, Heiss P, Senekowitsch-Schmidtke R, Schwaiger M, Stocklin G (1999) Synthesis and radiopharmacology of $O-(2-[18 \mathrm{~F}]$ fluoroethyl)-L-tyrosine for tumor imaging. J Nucl Med 40(1):205-212

6. Weber WA, Wester HJ, Grosu AL, Herz M, Dzewas B, Feldmann HJ, Molls M, Stocklin G, Schwaiger M (2000) $O$-(2-[18F]fluoroethyl)-L-tyrosine and L-[methyl-11C]methionine uptake in brain tumours: initial results of a comparative study. Eur J Nucl Med 27(5):542-549

7. Pauleit D, Floeth F, Herzog H, Hamacher K, Tellmann L, Muller HW, Coenen HH, Langen KJ (2003) Whole-body distribution and dosimetry of $O-(2-[18 \mathrm{~F}]$ fluoroethyl)-L-tyrosine. Eur $\mathrm{J}$ Nucl Med Mol Imaging 30(4):519-524. doi:10.1007/s00259-0031118-0

8. Popperl G, Kreth FW, Mehrkens JH, Herms J, Seelos K, Koch W, Gildehaus FJ, Kretzschmar HA, Tonn JC, Tatsch K (2007) FET PET for the evaluation of untreated gliomas: correlation of FET uptake and uptake kinetics with tumour grading. Eur J Nucl Med Mol Imaging 34(12):1933-1942. doi:10.1007/s00259-007-0534-y

9. Kunz M, Thon N, Eigenbrod S, Hartmann C, Egensperger R, Herms J, Geisler J, la Fougere C, Lutz J, Linn J, Kreth S, von Deimling A, Tonn JC, Kretzschmar HA, Popperl G, Kreth FW (2011) Hot spots in dynamic (18)FET-PET delineate malignant tumor parts within suspected WHO grade II gliomas. Neuro Oncol 13(3):307-316. doi:10.1093/neuonc/noq196

10. Spaeth N, Wyss MT, Weber B, Scheidegger S, Lutz A, Verwey J, Radovanovic I, Pahnke J, Wild D, Westera G, Weishaupt D, Hermann DM, Kaser-Hotz B, Aguzzi A, Buck A (2004) Uptake of $18 \mathrm{~F}$-fluorocholine, $18 \mathrm{~F}$-fluoroethyl-L-tyrosine, and 18F-FDG in acute cerebral radiation injury in the rat: implications for separation of radiation necrosis from tumor recurrence. $\mathbf{J}$ Nucl Med 45(11):1931-1938

11. Piroth MD, Pinkawa M, Holy R, Klotz J, Nussen S, Stoffels G, Coenen HH, Kaiser HJ, Langen KJ, Eble MJ (2011) Prognostic value of early [18F]fluoroethyltyrosine positron emission tomography after radiochemotherapy in glioblastoma multiforme.
Int J Radiat Oncol Biol Phys 80(1):176-184. doi:10.1016/ j.ijrobp.2010.01.055

12. Dunet V, Rossier C, Buck A, Stupp R, Prior JO (2012) Performance of 18F-fluoro-ethyl-tyrosine (18F-FET) PET for the differential diagnosis of primary brain tumor: a systematic review and metaanalysis. J Nucl Med 53(2):207-214. doi:10.2967/ jnumed.111.096859

13. Bossuyt PM, Reitsma JB, DE Bruns, Gatsonis CA, Glasziou PP, Irwig LM, Lijmer JG, Moher D, Rennie D, de Vet HC, Standards for Reporting of Diagnostic Accuracy (2003) Towards complete and accurate reporting of studies of diagnostic accuracy: the STARD Initiative. Radiology 226(1):24-28

14. Whiting P, Rutjes AW, Reitsma JB, Bossuyt PM, Kleijnen J (2003) The development of QUADAS: a tool for the quality assessment of studies of diagnostic accuracy included in systematic reviews. BMC Med Res Methodol 3:25. doi:10.1186/ 1471-2288-3-25

15. Rosman AS, Korsten MA (2007) Application of summary receiver operating characteristics (sROC) analysis to diagnostic clinical testing. Adv Med Sci 52:76-82

16. Egger M, Davey Smith G, Schneider M, Minder C (1997) Bias in meta-analysis detected by a simple, graphical test. BMJ 315(7109):629-634

17. Deeks JJ, Macaskill P, Irwig L (2005) The performance of tests of publication bias and other sample size effects in systematic reviews of diagnostic test accuracy was assessed. J Clin Epidemiol 58(9):882-893. doi:10.1016/j.jclinepi.2005.01.016

18. Floeth FW, Pauleit D, Sabel M, Reifenberger G, Stoffels G, Stummer W, Rommel F, Hamacher K, Langen KJ (2006) 18FFET PET differentiation of ring-enhancing brain lesions. J Nucl Med 47(5):776-782

19. Floeth FW, Pauleit D, Sabel M, Stoffels G, Reifenberger G, Riemenschneider MJ, Jansen P, Coenen HH, Steiger HJ, Langen KJ (2007) Prognostic value of $O$-(2-18F-fluoroethyl)-L-tyrosine PET and MRI in low-grade glioma. J Nucl Med 48(4):519-527

20. Pauleit D, Stoffels G, Bachofner A, Floeth FW, Sabel M, Herzog H, Tellmann L, Jansen P, Reifenberger G, Hamacher K, Coenen HH, Langen KJ (2009) Comparison of (18)F-FET and (18)F-FDG PET in brain tumors. Nucl Med Biol 36(7):779-787. doi:10.1016/ j.nucmedbio.2009.05.005

21. Pauleit D, Floeth F, Tellmann L, Hamacher K, Hautzel H, Muller HW, Coenen HH, Langen KJ (2004) Comparison of $O-(2-18 \mathrm{~F}-$ fluoroethyl)-L-tyrosine PET and 3-123I-iodo-alpha-methyl-Ltyrosine SPECT in brain tumors. J Nucl Med 45(3):374-381

22. Pichler R, Dunzinger A, Wurm G, Pichler J, Weis S, Nussbaumer K, Topakian R, Aigner RM (2010) Is there a place for FET PET in the initial evaluation of brain lesions with unknown significance? Eur J Nucl Med Mol Imaging 37(8):1521-1528. doi: 10.1007/s00259-010-1457-6

23. Calcagni ML, Galli G, Giordano A, Taralli S, Anile C, Niesen A, Baum RP (2011) Dynamic $O-(2-[18 \mathrm{~F}]$ fluoroethyl)-L-tyrosine (F18 FET) PET for glioma grading: assessment of individual probability of malignancy. Clin Nucl Med 36(10):841-847. doi: 10.1097/RLU.0b013e3182291b40

24. Jeong SY, Lim SM (2012) Comparison of $3^{\prime}$-deoxy-3'-[18F]fluorothymidine PET and $O-(2-[18 \mathrm{~F}]$ fluoroethyl $)$-L-tyrosine PET in patients with newly diagnosed glioma. Nucl Med Biol 39(7): 977-981. doi:10.1016/j.nucmedbio.2012.02.009

25. Weckesser M, Langen KJ, Rickert CH, Kloska S, Straeter R, Hamacher K, Kurlemann G, Wassmann H, Coenen HH, Schober O (2005) $O-(2-[18 \mathrm{~F}]$ fluorethyl)-L-tyrosine PET in the clinical evaluation of primary brain tumours. Eur J Nucl Med Mol Imaging 32(4):422-429. doi:10.1007/s00259-004-1705-8

26. Jansen NL, Graute V, Armbruster L, Suchorska B, Lutz J, Eigenbrod S, Cumming P, Bartenstein P, Tonn JC, Kreth FW, la 
Fougere C (2012) MRI-suspected low-grade glioma: is there a need to perform dynamic FET PET? Eur J Nucl Med Mol Imaging 39(6):1021-1029. doi:10.1007/s00259-012-2109-9

27. Pauleit D, Floeth F, Hamacher K, Riemenschneider MJ, Reifenberger G, Muller HW, Zilles K, Coenen HH, Langen KJ (2005) $O$-(2-[18F]fluoroethyl)-L-tyrosine PET combined with MRI improves the diagnostic assessment of cerebral gliomas. Brain 128(Pt 3):678-687. doi:10.1093/brain/awh399

28. Floeth FW, Sabel M, Ewelt C, Stummer W, Felsberg J, Reifenberger G, Steiger HJ, Stoffels G, Coenen HH, Langen KJ (2011) Comparison of (18)F-FET PET and 5-ALA fluorescence in cerebral gliomas. Eur J Nucl Med Mol Imaging 38(4):731-741. doi:10.1007/s00259-010-1690-z

29. Floeth FW, Pauleit D, Wittsack HJ, Langen KJ, Reifenberger G, Hamacher K, Messing-Junger M, Zilles K, Weber F, Stummer W, Steiger HJ, Woebker G, Muller HW, Coenen H, Sabel M (2005) Multimodal metabolic imaging of cerebral gliomas: positron emission tomography with [18F]fluoroethyl-L-tyrosine and magnetic resonance spectroscopy. J Neurosurg 102(2):318-327. doi: 10.3171/jns.2005.102.2.0318

30. Stockhammer F, Plotkin M, Amthauer H, van Landeghem FK, Woiciechowsky C (2008) Correlation of F-18-fluoro-ethyl-tyrosin uptake with vascular and cell density in non-contrastenhancing gliomas. J Neurooncol 88(2):205-210. doi:10.1007/ s11060-008-9551-3

31. Benouaich-Amiel A, Lubrano V, Tafani M, Uro-coste E, Gantet P, Sol JC, Roux F, Bousquet P, Julian A, Sabatier J, Tremoulet M, Cances-Lauwers V, Delisle MB, Clanet M, Esquerre JP, Payoux P (2010) Evaluation of $O$-(2-[18F]-fluoroethyl)-L-tyrosine in the diagnosis of glioblastoma. Arch Neurol 67(3):370-372. doi:10.1001/archneurol.2010.22

32. Plotkin M, Blechschmidt C, Auf G, Nyuyki F, Geworski L, Denecke T, Brenner W, Stockhammer F (2010) Comparison of F-18 FET-PET with F-18 FDG-PET for biopsy planning of non-contrast-enhancing gliomas. Eur Radiol 20(10):2496-2502. doi: 10.1007/s00330-010-1819-2

33. Lee TS, Ahn SH, Moon BS, Chun KS, Kang JH, Cheon GJ, Choi CW, Lim SM (2009) Comparison of 18F-FDG, 18F-FET and 18F-FLT for differentiation between tumor and inflammation in rats. Nucl Med Biol 36(6):681-686. doi:10.1016/j.nucmedbio. 2009.03.009

34. Laique S, Egrise D, Monclus M, Schmitz F, Garcia C, Lemaire C, Luxen A, Goldman S (2006) L-amino acid load to enhance PET differentiation between tumor and inflammation: an in vitro study on (18)F-FET uptake. Contrast Media Mol Imaging 1(5):212-220. doi:10.1002/cmmi.107

35. Chang CH, Wang HE, Wu SY, Fan KH, Tsai TH, Lee TW, Chang SR, Liu RS, Chen CF, Chen CH, Fu YK (2006) Comparative evaluation of FET and FDG for differentiating lung carcinoma from inflammation in mice. Anticancer Res 26(2A):917-925

36. Salber D, Stoffels G, Pauleit D, Oros-Peusquens AM, Shah NJ, Klauth P, Hamacher K, Coenen HH, Langen KJ (2007) Differential uptake of $O$-(2-18F-fluoroethyl)-L-tyrosine, L-3H- methionine, and 3H-deoxyglucose in brain abscesses. J Nucl Med 48(12):2056-2062. doi:10.2967/jnumed.107.046615

37. Pöpperl G, Götz C, Rachinger W, Gildehaus FJ, Tonn JC, Tatsch K (2004) Value of $O$-(2-[18F]fluoroethyl)-L-tyrosine PET for the diagnosis of recurrent glioma. Eur J Nucl Med Mol Imaging 31(11):1464-1470. doi:10.1007/s00259-004-1590-1

38. Möller-Hartmann W, Herminghaus S, Krings T, Marquardt G, Lanfermann H, Pilatus U, Zanella FE (2002) Clinical application of proton magnetic resonance spectroscopy in the diagnosis of intracranial mass lesions. Neuroradiology 44(5):371-381. doi: 10.1007/s00234-001-0760-0

39. Pöpperl G, Kreth FW, Herms J, Koch W, Mehrkens JH, Gildehaus FJ, Kretzschmar HA, Tonn JC, Tatsch K (2006) Analysis of 18F-FET PET for grading of recurrent gliomas: is evaluation of uptake kinetics superior to standard methods? J Nucl Med 47(3):393-403

40. Stadlbauer A, Prante O, Nimsky C, Salomonowitz E, Buchfelder M, Kuwert T, Linke R, Ganslandt O (2008) Metabolic imaging of cerebral gliomas: spatial correlation of changes in $O-(2-18 \mathrm{~F}-$ fluoroethyl)-L-tyrosine PET and proton magnetic resonance spectroscopic imaging. J Nucl Med 49(5):721-729. doi:10.2967/ jnumed.107.049213

41. Munck Af Rosenschöld P, Engelholm S, Ohlhues L, Law I, Vogelius I, Engelholm SA (2011) Photon and proton therapy planning comparison for malignant glioma based on CT, FDGPET, DTI-MRI and fiber tracking. Acta Oncol 50(6):777-783. doi:10.3109/0284186X.2011.584555

42. Herzog H, Langen KJ, Weirich C, Rota Kops E, Kaffanke J, Tellmann L, Scheins J, Neuner I, Stoffels G, Fischer K, Caldeira L, Coenen HH, Shah NJ (2011) High resolution BrainPET combined with simultaneous MRI. Nuklearmedizin 50(2):74-82. doi:10.3413/Nukmed-0347-10-09

43. Vander Borght T, Asenbaum S, Bartenstein P, Halldin C, Kapucu O, Van Laere K, Varrone A, Tatsch K, European Association of Nuclear Medicine (2006) EANM procedure guidelines for brain tumour imaging using labelled amino acid analogues. Eur J Nucl Med Mol Imaging 33(11):1374-1380. doi:10.1007/s00259-0060206-3

44. Langen KJ, Bartenstein $\mathrm{P}$, Boecker $\mathrm{H}$, Brust $\mathrm{P}$, Coenen $\mathrm{HH}$, Drzezga A, Grunwald F, Krause BJ, Kuwert T, Sabri O, Tatsch K, Weber WA, Schreckenberger M (2011) German guidelines for brain tumour imaging by PET and SPECT using labelled amino acids. Nuklearmedizin 50(4):167-173. doi:10.3413/nuk-2011041

45. Heinzel A, Stock S, Langen KJ, Müller D (2012) Cost-effectiveness analysis of amino acid PET-guided surgery for supratentorial high-grade gliomas. J Nucl Med 53(4):552-558. doi: 10.2967/jnumed.111.097352

46. Heinzel A, Stock S, Langen KJ, Muller D (2012) Cost-effectiveness analysis of FET PET-guided target selection for the diagnosis of gliomas. Eur $\mathbf{J}$ Nucl Med Mol Imaging 39(7):1089-1096. doi:10.1007/s00259-012-2093-0 